\title{
Using Humanized Mice to Evaluate the Pathogenesis of Variola Virus and Ebolavirus
}

Cynthia S. Goldsmith ${ }^{1}$, Christina L. Hutson ${ }^{2}$, Brian H. Bird ${ }^{3,4}$, Clifton P. Drew ${ }^{1,5}$, Victoria Olson ${ }^{2}$, Christina F. Spiropoulou ${ }^{3}$, Sherif R. Zaki ${ }^{1}$, and Jana M. Ritter ${ }^{1}$

${ }^{1 .}$ Infectious Diseases Pathology Branch, Centers for Disease Control and Prevention, Atlanta, USA

2. Poxvirus and Rabies Branch, Centers for Disease Control and Prevention, Atlanta, USA

3. Viral Special Pathogens Branch, Division of High-Consequence Pathogens and Pathology, Centers for Disease Control and Prevention, Atlanta, USA.

4. University of California Davis Veterinary School, Davis, CA, USA.

${ }^{5 .}$ Peninsular Veterinary Pathology Consulting, San Diego, CA, USA.

Highly immunodeficient mice can be engrafted with human immune cells and/or human fetal tissues in an effort to recapitulate the human immune system. So-called humanized mice have been instrumental in the elucidation of the pathogenesis of some human infectious diseases where non-human primates are the only other alternative or where rodent models can only be used with highly adapted viral isolates. Humanized mice have recently been used to study the pathogenesis of several pathogens, including Mycobacterium tuberculosis, Plasmodium falciparum, Salmonella enterica serovar Typhi, HIV, Kaposi's sarcoma-associated virus (HHV-8), hepatitis C, Epstein-Barr, dengue, and influenza viruses [1]. Two studies at the Centers for Disease Control and Prevention have used humanized mice infected with Variola virus (VARV), the causative agent of smallpox, or with Ebolavirus (EBOV), to better understand the pathogenesis of and immune response to these deadly viruses. In the United States, VARV and EBOV are select agents, subject to the select agent regulations (42 CFR part 73), and mice were housed and challenged with viruses in the CDC High Containment Laboratory (HCL). Once issue samples were collected, fixed, and gamma-irradiated, the carcasses were incinerated.

There has been a need for a better animal model of VARV because currently non-human primates are the only nonhuman animals to demonstrate overt disease. Humanized NSG, PBMC and BLT mice (The Jackson Laboratory) were challenged intranasally with VARV inoculum. While monitoring the mice for clinical criteria warranting euthanasia, high mortality with disseminated infection was seen beginning at day 13 for humanized NSG and BLT mice in a dose-dependent response, validating that humanized mice are a suitable animal model for human smallpox. EM studies found predominantly immature VARV particles in the murine-origin livers of the three animals studied, with few mature particles present (Fig. 1A, B). In contrast, the murine-origin uterus in a humanized PBMC mouse and the grafted human fetal thymic tissue from a BLT mouse showed mature as well as immature particles (Fig. 1C). These preliminary results warrant further investigation. Pathological review of infected mice found widespread tissue necrosis and IHC labeling of poxviral antigen in liver, adrenal gland, and reproductive and lymphoid tissues (Fig. 1 D, E).

Humanized BLT mice infected with a high-dose of the historical prototype EBOV (Mayinga 1976) showed severe ebola virus disease and uniform mortality. EM assessment confirmed viral replication, as shown by the production of viral nucleocapsids in hepatocytes and mature, extracellular particles in the sinusoidal space (Fig. 3 A, B). Histopathologic examination found the most severe pathologic findings in the liver, adrenal glands and perirenal/periadrenal soft tissue. Despite being of murine origin, hepatocytes produced large fibrillar or globular inclusions, typical of human EBOV infection, which stained by immunohistochemistry using EBOV antigenspecific mouse or rabbit polyclonal antibodies (Fig. 2 C, D). Similar to fatal human infections, infected mice developed high-level virus replication and elevated proinflammatory cytokine levels (also known as a "cytokine storm") [2].

The development and improvement of humanized mice has greatly enhanced the research into human infectious diseases that might not have otherwise had a suitable animal model. These mice can be used to study the human immunologic and biologic responses to experimental infection, and as a preclinical model for evaluation of 
therapeutic treatments.

References:

[1] N Walsh et al, Annu Rev Pathol 12 (2017), p. 187.

[2] BH Bird et al, J Infect Dis 213 (2016), p. 703.
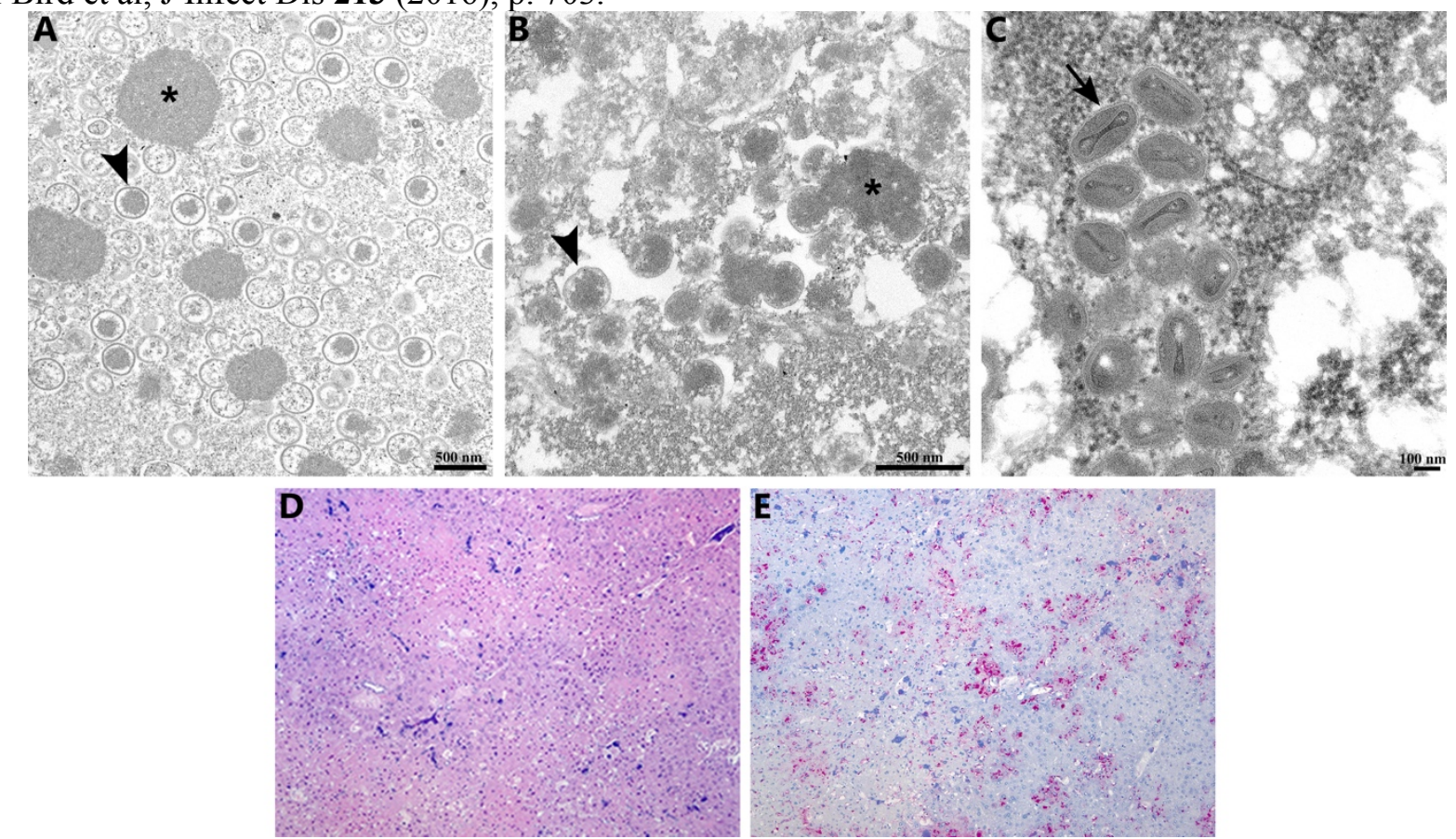

Figure 1. VARV humanized mice. A, B) Immature viral particles (arrowheads) and viroplasm (asterisks) within murine-origin hepatocytes. C) Intracellular mature virions within grafted human-origin thymus. D) H\&E of murine-origin liver showing areas of cell necrosis. E) Abundant immunostaining of VARV antigens in murine-origin liver. D, E - 10x original magnification.
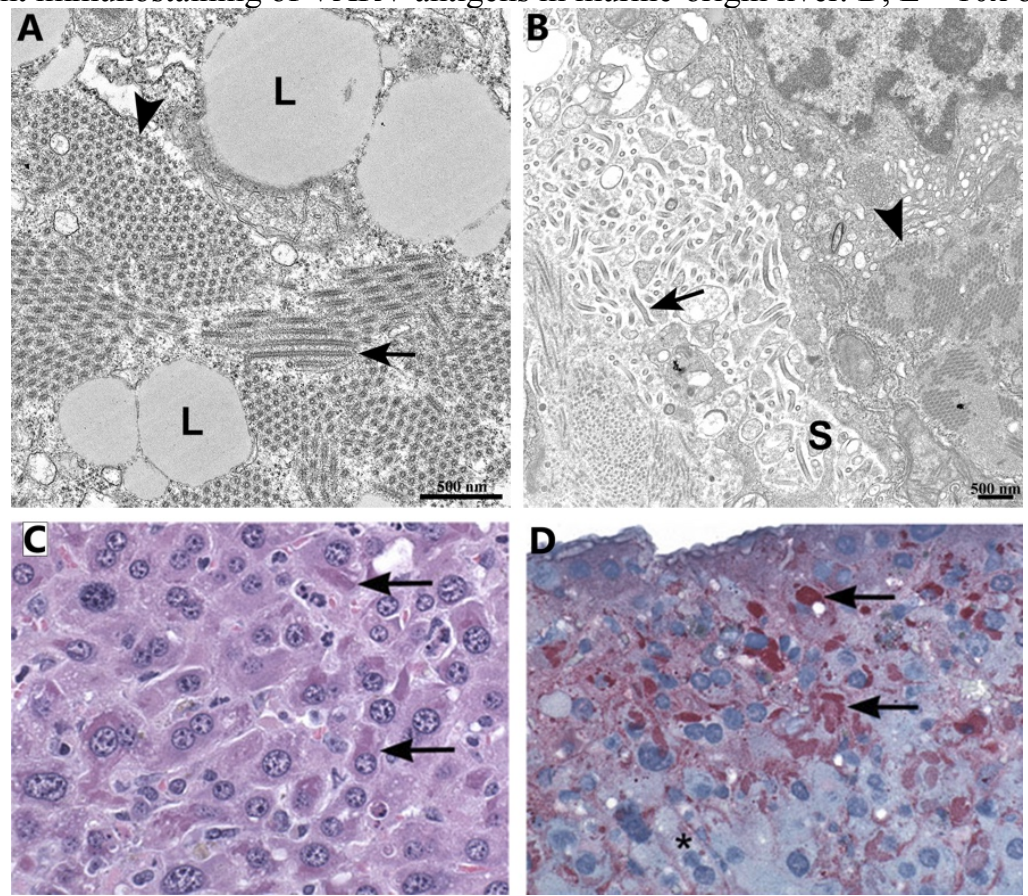

Figure 2. EBOV in livers of humanized mice. A) Filamentous viral nucleocapsids cut longitudinally (arrow) and in crosssection (arrowhead). L, lipid. B) Mature viral particles (arrow) are seen within the sinusoidal space (S), and a viral inclusion (arrowhead) containing nucleocapsids cut in cross-section is seen within a hepatocyte. C) H\&E staining showing characteristic eosinophilic filamentous inclusion bodies (arrows). D) Immunostaining of inclusion bodies (arrows) and sinusoidal lining cells (asterisk). C, D-400x original magnification. 